\title{
PERFIL EPIDEMIOLÓGICO DA HANSENÍASE NO ESTADO DO TOCANTINS NOPERÍODO DE 2015 A 2020
}

\section{EPIDEMIOLOGICAL PROFILE OF LEPROSY IN THE STATE OF TOCANTINS FROM 2015 TO 2020}

\author{
Luiza Moreira Cunha ${ }^{1}$ \\ Amadeu Martins Moreira Gonçalves ${ }^{2}$ \\ Rejanne Lima Arruda ${ }^{3}$
}

RESUMO: OBJETIVO: O presente estudo teve como objetivo analisar dados epidemiológicos da prevalência de hanseníase no Estado do Tocantins no período de 2015 a 2020. MÉTODO: Foi realizado um estudo epidemiológico descritivo com dados obtidos peloSistema de Informação de Agravos e Notificação (SINAN), tendo como sujeito de pesquisa a população residente no Tocantins. As variáveis analisadas foram: Prevalência, sexo, idade, localidade, escolaridade e tipo de saída. RESULTADOS: Foram registrados 9789 casos de hanseníase, sendo 5413(55,3\%) do sexo masculino e $4376(44,7 \%)$ do sexo feminino. A faixa etária mais acometida foi de 20 a 59 anos com 6577 (67,2\%), não teve relação direta com o nível de escolaridade e a quanto ao tipo de saída a maioria obteve cura. CONCLUSÃO: Os resultados demonstraram a gravidade dessa doença no Tocantins, que mesmo antiga, essa patologia continua sendo um sério problema de saúde pública e necessita de maior esclarecimento para a população quanto a transmissão, tratamento e prevenção, diminuindo, assim, o preconceito e levando a busca pelo tratamento adequado.

Palavras chave: Endemias; Doenças Infectocontagiosas; Perfil de Saúde; Saúde Pública.

ABSTRACT: OBJECTIVE: This study aims to analyze epidemiological data of leprosy prevalence from the Information System of Diseases aggravations and Notification (SINAN), in the period from 2015 to 2020. METHOD: A descriptive

\footnotetext{
1 Aluno de graduação do $7^{\circ}$ período do curso de Medicina do Centro Universitário Presidente Antônio Carlos da cidade de Araguaína-TO.

${ }^{2}$ Aluno de graduação do $8^{\circ}$ período do curso de Medicina do Centro Universitário Presidente Antônio Carlos da cidade de Araguaína-TO.

${ }^{3}$ Carlos da cidade de Araguaína-TO.

Professora Doutora do Centro Universitário Presidente Antônio Carlos da cidade de Araguaína-TO.
} 
epidemiological study was carried out with data obtained from Information System of Diseases aggravations and Notification (SINAN), with the population residing in Tocantins State, Brazil. The variables analyzed were: prevalence, sex, age, locality, education and evolution. RESULTS: The leprosy cases recorded were 9789, male were 5413 (55.3\%) and 4376 (44.70\%) were female. The age group most affected was 20 to 59 years with 6577 (67.2\%), These results are not directly linked to the level of education. The cure was obtained by the most of the population in Tocantins State, Brazil. CONCLUSION: The results showed the severity of this disease in Tocantins State, Brazil that even though it is an old disease, it continues to be a serious public health problem and needs to be better explained to the population regarding transmission, treatment, and prevention, this reducing the prejudice and leading to the search for adequate treatment.

Keywords: Endemics; Infectocontagious diseases; Health Profile; Public health. 


\section{INTRODUÇÃO}

Em 1873, quando a lepra ainda era vista como uma punição divina, o cientista norueguês Gerhard Henrik Armauer Hansen relacionou a doença com o microrganismo Mycobacterium leprae como o agente etiológico da hanseníase, a partir de biópsias de lesões cutâneas, confirmando a natureza infecciosa da enfermidade (TRABULSI; ALTERTHUM, 2008).

A hanseníase é uma patologia infecciosa crônica causada pelo Mycobacterium leprae. A preferência pela pele e nervos periféricos dá características peculiares a esta doença, tornandoo seu diagnóstico simples na maioria dos casos. Em contraponto, o dano neurológico responsabiliza-se pelas sequelas que podem surgir. Constitui importante problema de saúde pública no Brasil e em vários países do mundo. Apesar de todo o empenho em sua eliminação, o Brasil continua sendo o segundo país em número de casos no mundo (ARAÚJO, 2003).

O período de incubação da doença varia de 2 a 7 anos, possui alta taxa de infecção, porém com baixa virulência (ALVES et al., 2010).

O Mycobacterium leprae pertence a ordem Actinomycelalis e família Mycobaderiaceae. Quanto a morfologia, tem a forma de bacilo reto ou levemente arqueado, com extremidades arredondadas, seu tamanho varia de 1 a $8 \mathrm{~mm}$ de comprimento e 0,3 $\mathrm{mm}$ de diâmetro, apresenta- se como intracelular obrigatório e infecta com maior frequência macrófagos (OPROMOLLA et al., 2000).

A transmissão do parasita ocorre por meio de uma pessoa doente, sem estar em tratamento, que elimina o bacilo pelas vias aéreas superiores para o meio externo, ocorrendo a transmissão para pessoas suscetíveis (NUNES; OLIVEIRA; VIEIRA, 2011).

Os sinais mais relevantes que indicam a hanseníase são as manchas, podendo ser homopigmentadas, vermelhas ou amarronzadas, planas ou elevadas, podendo aparecer em qualquer parte do corpo. Pode apresentar também manchas com perda de sensibilidade, atrofias, paresias e paralisias musculares que, se não 
tratadas adequadamente, podem evoluir para incapacidades físicas permanentes (EIDT, 2004).

As manifestações clinicas da doença estão diretamente direcionadas ao tipo de respostaimunológica ao $M$. Leprae. Tem-se então, as seguintes formas clinicas da doença: a Hanseníase indeterminada (HI) que ocorre após o período de incubação, caracterizada pelo aparecimento de manchas hipocrômicas na pele, com alterações na sensibilidade. A Hanseníase Tuberculóide (HT), caracterizada por lesões bem delimitadas, hiposensíveis, com número reduzido e distribuição assimétrica. A Hanseníase Virchoviana (HV), que é a forma multibacilar, aconteceprincipalmente em imunodeprimidos e, nesta apresentação da doença, ocorre o aparecimento de pápulas, máculas e nódulos. Na Hanseníase Dimorfa (HD) ocorre uma instabilidade imunológica, fazendo com que haja uma variação em suas manifestações clínicas: as lesões da pele são numerosas e sua morfologia mescla aspectos de HV e HT, podendo haver predominância ora de um, ora de outro (ARAÚJO, 2003).

Além dos sinais e sintomas supracitados, têm-se febre e artralgia, associados a caroços dolorosos, de aparecimento súbito; surgimento repentino de manchas dormentes com dor nos nervos dos cotovelos, joelhos e tornozelos; entupimento, feridas e ressecamento do nariz; sensação de secura e areia nos olhos; dor, choque e/ou espessamento de nervos periféricos; diminuição e/ou perda de sensibilidade nas áreas dos nervos afetados, principalmente nos olhos, mãos e pés; diminuição e/ou perda de força nos músculos inervados por estes nervos, principalmente nos membros superiores e inferiores; edema de mãos e pés com cianose (arroxeamento dos dedos) e ressecamento da pele (BRASIL, 2002).

O diagnóstico da hanseníase é feito de forma clínica e laboratorialmente. $\mathrm{O}$ diagnóstico clínico é feito através de uma boa anamnese, onde se faz um histórico detalhado e busca pelas condições de vida desse paciente. Faz-se o exame dermatoneurológico, no intuito de identificarlesões ou áreas da pele com alterações de sensibilidade e/ou comprometimento neural periférico. Já o diagnóstico laboratorial se dá por meio da baciloscopia. (BRASIL, 2009).

A baciloscopia é um exame microscópico, onde se usa um esfregaço de raspados de tecido dérmico, colhido nas áreas suspeitas e também nos lóbulos das orelhas, nos cotovelos e joelhos, observando a presença do bacilo. O resultado 
apresenta-se sob forma de índice baciloscópio (IB), com uma variação de 0 a 6+. A baciloscopia mostra-se negativa $(I B=0)$ nas formas tuberculóide e indeterminada, fortemente positiva na forma virchoviana e, revela um resultado variável na forma dimorfa (ARAÚJO, 2003).

O tratamento da Hanseníase no Brasil foi padronizado pelo Ministério da Saúde seguindo padrões da OMS. Os esquemas de poliquimioterapias (PQT) é composta pela associação de dapsona, clofazimina e rifampicina, e altera-se de acordo com a classificação do paciente (adulto ou pediátrico) e da manifestação apresentada (paucibacilar ou multibacilar). Isso deve ser seguido rigorosamente para o sucesso da terapia em, relativamente, pouco tempo (CURTO; BARBOZA; PASCHOAL, 2007; PEREIRA et al., 2008; FERREIRA et al., 2010; LIMA et al., 2010).

A região Norte do Brasil é a que apresenta as maiores taxas de prevalência e concentra a maior parte dos casos confirmados de hanseníase (EIDT, 2004). No Estado do Tocantins, essaenfermidade é considerada hiperendêmica, de acordo com os rankings do Ministério da Saúde. Em 2012, o estado ocupou o segundo lugar no ranking brasileiro (BRASIL, 2013).

Diante dos fatos mencionados e da necessidade de atualização das pesquisas nesta área, fica por objetivo deste artigo analisar os dados epidemiológicos da prevalência de hanseníase no Estado do Tocantins, no período de 2015 a 2020.

\section{MÉTODOLOGIA}

O Tocantins é um estado brasileiro localizado na Região Norte, dividido em 139 cidades, ocupando uma área de $277.621 \mathrm{~km}^{2}$. O estado é separado em oito regiões de saúde com base na cidade, população e densidade populacional para oferecer ações e serviços mínimos para cada região. Em 2020, a população estimada foi de 1.590,248 milhões de pessoas, um aumento de 18,4\% desde 2000 (MONTEIRO et al, 2015). 
O presente estudo aborda uma pesquisa descritiva epidemiológica de análise quantitativa. Os dados usados foram coletados de fichas do Sistema de Informação e Agravos de Notificação (SINAN), presentes no DataSUS. Tais informações foram referentes à incidência de casos de hanseníase no referido estado, entre os anos de 2015 e 2020. Sendo assim, tal trabalho dispensa o uso de Termo de Consentimento Livre e Esclarecido por não fazer uso de amostras biológicas, sendo utilizados dados coletados em fichas epidemiológicaspresentes no DataSUS.

Para analisar dos dados, foram organizados por meio do programa Microsoft Excel / 2013 gráficos e tabelas com as seguintes variáveis: Prevalência, sexo, idade, localidade, escolaridade e tipos de saída. Mediante a análise de tais dados, foram construídos os resultados, discussão e conclusão.

\section{RESULTADOS}

Segundo dados cedidos pelo DataSUS, no Sistema de Informação e agravos de Notificação (SINAN), o Brasil, nos anos de 2015 a 2020, notificou 198.478 casos de hanseníase. Nesse páreo, em números absolutos, o Mato Grosso é o estado com maior o números de casos, com 26.401, seguido pelo Maranhão com 24.055, já o Tocantins está em oitavo com 9.789 casos.

No estado do Tocantins, ao analisar os dados, do total de 9789 (100\%) das notificações, percebeu-se uma oscilação significativa entre os anos de 2015 e 2018, saindo de 1071 (10,9\%)casos no ano de 2015, 1731 (17,7\%) em 2016, 1685(17,2\%) em 2017, para 2155 (22\%) notificações em 2018, ano esse com a maior prevalência entre os dados analisados. Logo, no ano de 2020 foram notificados $1113(11,4 \%)$ casos, havendo redução significativa em relação ao ano de 2019 que verificou-se $2034(20,8 \%)$ (Gráfico 1). 
Gráfico 1: Casos de Hanseníase no Estado do Tocantins no período de 2015 a 2020.

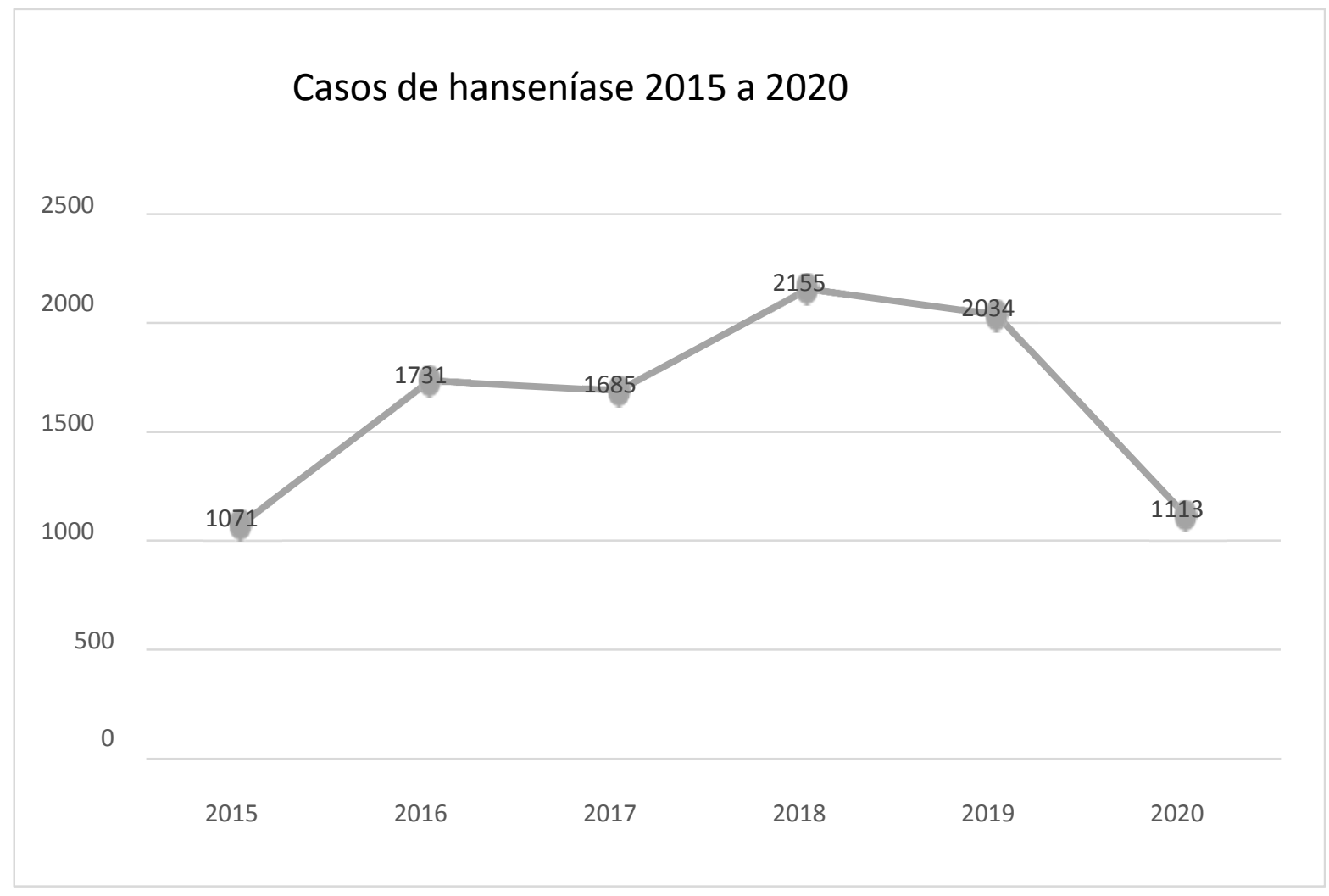

Fonte: Sistema de Informação de Agravos e Notificações (SINAN) /2021.

Foram analisados 9789 casos de hanseníase, notificados ao Sistema de Informação de Agravos de Notificação (SINAN) entre os anos de 2015 a 2020, no Estado do Tocantins, dos quais $5413(55,3 \%)$ do sexo masculino e $4376(44,70 \%)$ do sexo feminino (Tabela 1).

Obteve-se, em todos os anos analisados, uma maior prevalência da Hanseníase nosexo masculino, em 2015 com 649 casos (60,6\%), 2016 com 943 (54,5\%), 2017 com 945 (56,1\%), 2018 com 1136 (52,7\%), 2019 com 1100 (54,1\%) e em 2020 com 640 (57,5\%). Em contrapartida, a prevalência entre o sexo feminino foi de $422(39,4 \%)$ em 2015, 788 (45,5\%) em 2016, 740 (43,9\%) em 2017, 1019 (47,3\%) em 2018, 934 (45,9\%) em 2019 e 473 (42,5\%) em 2020 (Tabela 1).

A faixa etária mais atingida pela Hanseníase em todos os anos analisados foi a de 20 a 59 anos, totalizando 67,2\% dos casos nos 6 anos. Em 2015 obteve 65,5\% 
das notificações, em 2016 teve 66,7\%, em 2017 teve 67,3\%, em 2018 teve 67,2\%, em 2019 teve $68,1 \%$ e em 2020 com 67,7\%. Já a faixa etária menos acometida, nos anos analisados, foram os de idade entre 1 a 19 anos, totalizando 11,3\% em todos anos estudados. Em 2015 atingiu 13,3\%, em 2016 teve 11,4\%, em 2017 obteve 10,4\%, em 2018 teve 11,3\%, em 2019 atingiu 11,6\% e, por fim, em 2020 teve 10,2\% (Tabela 1).

Tabela 1: Prevalência de casos de Hanseníase com dados sociodemográficos no período de 2015 a 2020 no Tocantins.

\begin{tabular}{|c|c|c|c|c|c|c|c|}
\hline & 2015 & 2016 & 2017 & 2018 & 2019 & 2020 & TOTAL \\
\hline \multicolumn{8}{|c|}{ SEXO } \\
\hline Masculino & $\begin{array}{c}649 \\
(60,6 \%)\end{array}$ & $\begin{array}{c}943 \\
(54,5 \%)\end{array}$ & $\begin{array}{c}945 \\
(56,1 \%)\end{array}$ & $\begin{array}{c}1136 \\
(52,7 \%)\end{array}$ & $\begin{array}{c}1100 \\
(54,1 \%)\end{array}$ & $\begin{array}{c}640 \\
(57,5 \%)\end{array}$ & $\begin{array}{c}5413 \\
(55,3 \%)\end{array}$ \\
\hline Feminino & $\begin{array}{c}422 \\
(39,4 \%)\end{array}$ & $\begin{array}{c}788 \\
(45,5 \%)\end{array}$ & $\begin{array}{c}740 \\
(43,9 \%)\end{array}$ & $\begin{array}{c}1019 \\
(47,3 \%)\end{array}$ & $\begin{array}{c}934 \\
(45,9 \%)\end{array}$ & $\begin{array}{c}473 \\
(42,5 \%)\end{array}$ & $\begin{array}{c}4376 \\
(44,7 \%)\end{array}$ \\
\hline \multicolumn{8}{|c|}{ FAIXA ETÁRIA } \\
\hline 1 a 19 anos & $\begin{array}{c}143 \\
(13,3 \%)\end{array}$ & $\begin{array}{c}197 \\
(11,4 \%)\end{array}$ & $\begin{array}{c}176 \\
(10,4 \%)\end{array}$ & $\begin{array}{c}243 \\
(11,3 \%)\end{array}$ & $\begin{array}{c}235 \\
(11,6 \%)\end{array}$ & $\begin{array}{c}114 \\
(10,2 \%)\end{array}$ & $\begin{array}{c}1108 \\
(11,3 \%)\end{array}$ \\
\hline 20 a 59 anos & $\begin{array}{c}701 \\
(65,5 \%)\end{array}$ & $\begin{array}{c}1155 \\
(66,7 \%)\end{array}$ & $\begin{array}{c}1134 \\
(67,3 \%)\end{array}$ & $\begin{array}{c}1448 \\
(67,2 \%)\end{array}$ & $\begin{array}{c}1386 \\
(68,1 \%)\end{array}$ & $\begin{array}{c}753 \\
(67,7 \%)\end{array}$ & $\begin{array}{c}6577 \\
(67,2 \%)\end{array}$ \\
\hline $\begin{array}{l}60 \text { ou mais } \\
\text { anos }\end{array}$ & $\begin{array}{c}227 \\
(21,2 \%)\end{array}$ & $\begin{array}{c}379 \\
(21,9 \%)\end{array}$ & $\begin{array}{c}375 \\
(22,3 \%)\end{array}$ & $\begin{array}{c}464 \\
(21,5 \%)\end{array}$ & $\begin{array}{c}413 \\
(20,3 \%)\end{array}$ & $\begin{array}{c}246 \\
(22,1 \%)\end{array}$ & $\begin{array}{c}2104 \\
(21,5 \%)\end{array}$ \\
\hline $\begin{array}{l}\text { TOTAL } \\
\text { anual }\end{array}$ & $\begin{array}{c}1071 \\
(10,9 \%)\end{array}$ & $\begin{array}{c}1731 \\
(17,7 \%)\end{array}$ & $\begin{array}{c}1685 \\
(17,2 \%)\end{array}$ & $\begin{array}{l}2155 \\
(22 \%)\end{array}$ & $\begin{array}{c}2034 \\
(20,8 \%)\end{array}$ & $\begin{array}{c}1113 \\
(11,4 \%)\end{array}$ & $\begin{array}{c}9789 \\
(100 \%)\end{array}$ \\
\hline
\end{tabular}

Fonte: Sistema de Informação de Agravos e Notificação (SINAN) /2021.

Entre os 9879 casos notificados no período de 2015 a 2020, 9690 haviam especificado o município de notificação. Desses 9690 (100\%), os municípios com maior número denotificações de hanseníase nos anos analisados foram, em primeiro lugar, a capital do estado, Palmas com 3968 (40,9\%) notificações, em segundo está Araguaína com 960 (9,9\%), em terceiro está Porto Nacional com 484 (5\%), em quarto está Gurupi com 437 (4,5\%), em quinto está Paraíso do Tocantins com 244 $(2,5 \%)$, em sexto está Miracema do Tocantins com $157(1,6 \%)$, em sétimo está Colinas do Tocantins com $136(1,4 \%)$ casos, em oitavo está Santa Fé do Araguaia 
com 125 (1,3\%), em nono está São Valério com $113(1,2 \%)$ e por fim em décimoestá Lagoa da confusão com $112(1,2 \%)$ casos reportados. Os demais 2954 (30,5\%) das notificações são de outros municípios do estado.

Quanto à escolaridade dos acometidos pela hanseníase, dos 9789 casos registrados, somente 7933 haviam informações sobre o nível escolar. Desses 7933 (100\%), 678 (8,5\%) eram analfabetos, 1554 (19,6\%) tinham $1^{\text {a }}$ a $4^{a}$ série do ensino fundamental incompleto, $513(6,5 \%)$ tinham $4^{\text {a }}$ série do ensino fundamental completa, $1339(16,9 \%)$ tinham $5^{\mathrm{a}}$ a $8^{\mathrm{a}}$ série do ensino fundamental incompleta, 713 (9\%) tinham ensino fundamental completo, 760 (9,6\%) tinham ensino médio incompleto, 1675 (21,1\%) tinham ensino médio completo, 197 (2,5\%) tinham educação superior incompleta e 504 (6,3\%) tinham educação superior completa.

Tendo em vista o tipo de saída, ou seja, a resolução ou evolução das notificações, entre os 9789 casos de hanseníase, vê-se que 2009 notificações não houveram informações quanto ao desfecho do episódio, tendo 7780 casos informando quanto à evolução. Desses 7780 (100\%), nota-se que a maioria obteve cura, contando com 6054 (77,8\%); 506 (6,5\%) abandonaram o tratamento; 487 $(6,2 \%)$ foram transferidos para outro município do Tocantins; $109(1,4 \%)$ foram transferidos para o mesmo município; 309 (4\%) foram transferidos para outro estado; $7(0,1 \%)$ foram transferidos para outro país; $138(1,8 \%)$ evoluiram para óbito; e 170 $(2,2 \%)$ foramerros diagnósticos.

\section{DISCUSSÃO}

Ao analisar os dados, foi possível detectar oscilações significativas, principalmente, nosanos de 2018 a 2020, podendo ser explicado por possíveis ações de controle da doença no estadoe municípios. O que também pode ser influenciado por ações educativas aplicadas no local e a capacitação de profissionais de saúde para realizarem o diagnóstico da doença de maneira eficiente, adequada e precoce (OMS, 2006). 
Através do presente estudo pode-se identificar a predominância dos casos de hanseníase em indivíduos do sexo masculino. Este perfil epidemiológico pode ser explicado pelo fato de estes apresentarem maior exposição a ambientes de risco, além disso, o fato dos homens, de maneira geral, apresentarem menor preocupação em relação a estética acaba dificultando o diagnóstico (SILVA et al., 2010). Além disso, os homens tem maior contato interpessoal no trabalho, menor preocupação com seu bem-estar físico e falta de acesso aos serviços médicos, o que torna esse grupo mais propício a doenças infecciosas e outros agravos. Isso sugere que a as condições de vida mais ativa do homem e o descaso com a saúde torna-os mais expostos a hanseníase (MOREIRA et al., 2014; BARRETO et al., 2014).

Tendo em vista a faixa etária dos casos supracitados, infere-se que a população mais afetada pela hanseníase é a economicamente ativa, mais especificamente dos 20 aos 59 anos, o que pode interferir significativamente na economia do estado, haja vista que os indivíduos dessa faixa etária podem sofrer mais danos como incompetências, lesões, estados reacionais, abdicarde atividades produtivas e gerar custos sociais e econômicos elevados (LANA et al., 2004 e ARAÚJO, 2003).

Observando a escolaridade, apesar do grupo majoritário ser dos que possuem Ensino Médio completo, nota-se a importância de se realizar a educação em saúde da população, pois revelou-se que $51,5 \%$ dos avaliados não haviam completado o Ensino Fundamental. Desse modo, um estudo de Minas Gerais sinaliza que essa falta de estudo pode dificultar a apreensãodas orientações sobre o tratamento e cuidados necessários para a prevenção e identificação dos sintomas (MIRANZI et al., 2010). Outrossim, essa informação é de grande importância para o planejamento das ações de educação em saúde e sensibilização da comunidade, pois pesquisassugerem que os profissionais de saúde se atentem quanto ao grau de escolaridade da populaçãodo território da abrangência de ação da unidade de saúde ao planejarem as atividades de prevenção de doenças e promoção de saúde, com o objetivo de garantir o entendimento da mensagem transmitida (AMARAL, 2008).

As regiões de saúde de Palmas, Araguaína e Gurupi apresentaram maior detecção no período, provavelmente por comporem as áreas de maior densidade populacional do estado (IBGE, 2020), bem como pela disponibilidade de uma rede 
de serviços de referência e contrareferência dando melhor suporte diagnóstico por profissionais especializados.

Quanto ao tipo de saída, nota-se que a maioria dos casos evoluíram para cura, o que demonstra boa adesão ao tratamento. No entanto, houveram $6,5 \%$ dos casos onde houve abandono do tratamento, o que pode levar a sequelas importantes ao acometido, podendo até mesmo incapacitá-lo. Dessa forma, como sugere pesquisas, somente $1 \mathrm{em}$ cada 3 dos portadores de hanseníase está notificado e que, dentre esses, muitos fazem um tratamento irregular ou o abandonam, tendo como consequência bacilos resistentes às medicações e que podem levar a dificuldades no tratamento da doença e agravamento da forma clínica, aumentando o problema de controle da hanseníase (MIRANZI et al., 2010). Vale ressaltar, que o tratamento adequado da doença é eficiente para prevenção e cura da mesma (FAC, 2011).

Estudos sugerem que, profissionais capazes de identificar sinais e sintomas dermatológicos, devem realizar busca ativa na população, no intuito de fazer diagnósticos mais precoces. Tais profissionais são, por exemplo: médicos, enfermeiros, odontólogos e agentes comunitários de saúde (LANZA et al., 2011).

Sendo assim, como sinalizam estudos, vê-se que a hanseníase no Brasil trata-se de uma situação crítica que necessita de análise cuidadosa para uma intervenção eficaz. Pois, tem distribuição heterogênia e reproduz as desigualdades socioeconômicas sociais, auxiliando a sua propagação principalmente quando associada à precariedade nas condições sanitárias (CORRÊA et al., 2012).

\section{CONCLUSÃO}

Pela observação dos aspectos analisados pôde-se traçar um perfil epidemiológico de prevalência de hanseníase no Estado Tocantins entre os anos de 2015 a 2020, através de dadoscoletados no SINAN. O Tocantins é o oitavo Estado brasileiro mais acometido em número de casos pela doença no período pesquisado, o que coloca o estado como um dos principais em incidência, por ter uma população 
menos numerosa em relação aos outros estados que também possuem alto número de casos. Essa patologia continua sendo um importante problema de saúdepública no estado e sua disseminação continua sendo ativa e contínua. Foi observado um número acentuado de casos de hanseníase até o ano de 2020, o que levanta a necessidade de ações intensivas de esclarecimento sobre a doença para que toda a população saiba como ocorreo contágio, tratamento e prevenção, diminuindo assim, o preconceito e, em contraponto, aumentar a busca pelo tratamento adequado.

Outro fator de grande importância é o fato da hanseníase ser uma doença estigmatizada. A falta de conhecimento da população dificulta a aceitação, até mesmo dos portadores, que abandonam ou se recusam a realizar o tratamento, além de omitirem que possuem a enfermidade (MIRANZI et al., 2010). Tal fato, acentua a necessidade de abordagem sobre a doença com a população, como anteriormente discutida.

\section{REFERÊNCIAS BIBLIOGRÁFICAS}

ALVES, Cinthia Janine Meira et al. Evaluation of the degree of incapacity of patients with a diagnosis of leprosy at a dermatology service in the state of São Paulo. Revista da Sociedade Brasileira de Medicina Tropical, v. 43, n. 4, 2010.

AMARAL, Evaldo Pinheiro; LANA, Francisco Carlos Félix. Análise espacial da Hanseníase na microrregião de Almenara, MG, Brasil. Revista Brasileira de Enfermagem, v. 61, n. SPE, p. 701-707, 2008.

ARAÚJO, Marcelo Grossi. Hanseníase no brasil. Revista da sociedade brasileira de medicina tropical, v. 36, n. 3, p. 373-382, 2003.

BARRETO, Josafá Gonçalves et al. Spatial analysis spotlighting early childhood leprosy transmission in a hyperendemic municipality of the Brazilian Amazon region. PLoS neglected tropical diseases, v. 8, n. 2, p. e2665, 2014.

BRASIL, 2009. GUIA DE VIGILÂNCIA EPIDEMIOLÓGICA. Secretaria de Vigilância em Saúde /MS. Caderno 07. Brasília-DF, 2009. Acesso em 23 de fevereiro de 2021.

BRASIL. Ministério da Saúde. Secretaria de Políticas de Saúde. Departamento de Atenção Básica. Guia para o controle da hanseníase. $1^{a}$ edição. Brasília, 2002.

BRASIL. Ministério da Saúde. Coordenação de Doenças Transmissíveis e Não Transmissíveis. Secretaria de Estado da Saúde do Tocantins. Boletim Epidemiológico de Hanseníase, 2013. Disponível em: http://www.portal.saude.gov.br.

CORRÊA, Rita da Graça Carvalhal Frazão et al. Epidemiological, clinical, and operational aspects of leprosy patients assisted at a referral service in the state of Maranhão, Brazil. Revista 
da Sociedade Brasileira de Medicina Tropical, v. 45, n. 1, p. 89-94, 2012.

CURTO, Márcio; BARBOZA, Denise B.; PASCHOAL, Vânia DA. Avaliação da importância do diagnóstico e tratamento precoce da hanseníase em relação ao custo do tratamento. Arq. ciênc. saúde, v. 14, n. 3, p. 153-160, 2007.

EIDT, Letícia Maria. Breve história da hanseníase: sua expansão do mundo para as Américas,o Brasil e o Rio Grande do Sul e sua trajetória na saúde pública brasileira. Saúde e sociedade, v. 13, n. 2, p. 76-88, 2004.

FERREIRA, Silvana Margarida Benevides et al. Recidivas de casos de hanseníase no estado de Mato Grosso. Revista de Saúde Pública, v. 44, p. 650-657, 2010. IBGE - INSTITUTO BRASILEIRO DE GEOGRAFIA E ESTATÍSTICA. Censo

Brasileiro de 2010. Rio de Janeiro IBGE, 2020.

LANA FCF, et al. Estimativa da prevalência oculta da hanseníase no Vale do Jequitinhonha: Minas Gerais. Rev Min Enferm; 8: 295-300, 2004.

LANZA, Fernanda Moura et al. Ações de controle da hanseníase: tecnologias desenvolvidas nos municípios do Vale do Jequitinhonha, Minas Gerais. Revista de Enfermagem do CentroOeste Mineiro, 2011.

LIMA, Hívena Maria Nogueira et al. Perfil epidemiológico dos pacientes com hanseníase atendidos em Centro de Saúde em São Luís, MA. Rev Bras Clin Med, v. 8, n. 4, p. 323-7, 2010.

MIRANZI, Sybelle de Souza Castro; PEREIRA, Lívia Helena de Morais; NUNES, Altacílio Aparecido. Perfil epidemiológico da hanseníase em um município brasileiro, no período de 2000 a 2006. Revista da Sociedade Brasileira de Medicina Tropical, v. 43, n. 1, p. 62-67, 2010.

MONTEIRO, L. L. et al. Tendências da hanseníase no Tocantins, um estado hiperendêmico do Norte do Brasil, 2001-2012. Cad. Saúde Pública. Vol 31. Núm. 5. 2015. Disponível em: $<$ https://www.scielosp.org/article/csp/2015.v31n5/971-

980/\#: :text=A\%20regi\%C3\%A3o\%20de\%20sa\%C3\%BAde\%20de\%20Gurupi\%20apresent ou\%20a\%20maior\%20ocorr\%C3\%AAncia,536\%3B\%203\%2C7\%25>. Acesso em:23/fev/2021.

MOREIRA, Renata Lívia Silva Fonsêca; FONTES, Wilma Dias de; BARBOZA, Talita Maia. Dificuldades de inserção do homem na atenção básica a saúde: a fala dos enfermeiros. Escola Anna Nery, v. 18, n. 4, p. 615-621, 2014.

NUNES, Joyce Mazza; OLIVEIRA, Eliany Nazaré; VIEIRA, Neiva Francenely Cunha. Hanseníase: conhecimentos e mudanças na vida das pessoas acometidas. Ciência \& Saúde Coletiva, v. 16, p. 1311-1318, 2011.

ORGANIZAÇÃO MUNDIAL DA SAÚDE. Estratégia global aprimorada para redução adicional da carga da hanseníase: período do plano: 2011-2015. Brasília: Organização Mundial da Saúde; 2006.

PEREIRA JUNIOR, FAC. Motivos do abandono ou interrupção do tratamento da hanseníase: uma revisão sistemática da literatura. Monografia (Gestão de saúde coletiva) Fundação Oswaldo Cruz. 2011.

PEREIRA, Sandra Valéria Martins et al. Avaliação da hanseníase: relato de experiência de acadêmicos de enfermagem. Revista brasileira de enfermagem, v. 61, n. SPE, p. 774-780, 2008.

SILVA, Antonio Rafael da et al. Hanseníase no Município de Buriticupu, Estado do Maranhão: busca ativa de casos na população adulta. Revista da Sociedade Brasileira de Medicina 
Tropical, v. 43, n. 6, p. 691-694, 2010.

TRABULSI, Luiz Rachid; ALTERTHUM, Flavio. Microbiologia. In: Microbiologia. 2005. p.718718.

URA, S.; OPROMOLLA, D. V. A. Noções de hansenologia. Bauru: Centro de Estudos Dr Reynaldo Quagliato, 2000. 